\title{
Relación entre empoderamiento familiar y calidad de los servicios de atención temprana
}

\section{Early Intervention Quality \& Family’s Empowerment}

\author{
Rosa Fernández Valero *, Ana Maria Serrano **, Robert Alexander McWilliam***, Margarita Cañadas**** \\ *Universidad de Minho, ** Universidad de Minho, *** Universidad de Alabama, ****Universidad Católica de Valencia
}

\begin{abstract}
Resumen
Este trabajo pretende estudiar el nivel de empoderamiento de las familias en relación con la Calidad de los Servicios de Atención Temprana en España, donde en la actualidad se está viviendo una transformación en los modelos de intervención. La recogida de datos se esta llevando a cabo a través de la percepción de las familias receptoras de estos servicios. Se están utilizando las siguientes herramientas: FOS (Early Childhood Outcomes Center, 2010); FES (Paul E. Koen, Neal DeChillo y Barbara J. Fieson, 1992), FSS (Dunst, Trivette y Jenkins, 1984) y Family FINESSE (McWilliam, 2015). Para llevar a cabo este trabajo, hemos desarrollado el Análisis Factorial Exploratorio de Family FINESSE. Así pues, presentamos los factores analizados en dicha escala, así como otros datos psicométricos de la misma.

Palabras clave: Empoderamiento Familiar, Atención Temprana, Análisis Factorial Exploratorio
\end{abstract}

\begin{abstract}
This paper pretend to study the level of empowerment of families in relation to the Quality of Early Intervention Services in Spain, where a transformation is currently underway in intervention models. The collection of data is being carried out through the perception of the families receiving these services. The following tools are being used: FOS (Early Childhood Outcomes Center, 2010); FES (Paul E. Koen, Neal DeChillo and Barbara J. Fieson, 1992), FSS (Dunst, Trivette and Jenkins, 1984) and Family FINESSE (McWilliam, 2015). To carry out this work, we have developed the Exploratory Factorial Analysis of Family FINESSE. Thus, we present the factors analyzed in this scale, as well as other psychometric data of the same. Keywords: Family Empowerment, Early Intervention, Exploratory Factorial Analysis
\end{abstract}

La familia y como apoyarlos, ha sido un constante interés en atención temprana desde sus inicios, pero no fue hasta los años ochenta y noventa del siglo pasado que en algunos países (USA, Países Nordicos) le dan importancia a roles de la familia más significativos en la atención temprana (Mc William, 2010; Espe-Sherwindt, 2008).

Probablemente este enfoque de atención centrada en la familia, como indicó Law et al. (2003), uno de los mas importantes precursores fue Carl Rogers (1961) quien con su modelo "centrado en el cliente" buscaba dar mayor importancia y control al propio cliente y no tanto al profesional. Fue en los años 60 cuando la "Association for the Care of Children in Hospital" comenzó a incorporar su ideas en el cuidado de los niños y sus familias subrayando la expression "cuidado centrado en la familia". Mas tarde aparecieron otros conceptos y contribuciones tal y como lo entendemos ahora, como atención temprana centrada en la familia. Dunst y Trivette (2009) señalan que los componentes clave de la construcción de este paradigma, vienen del acercamiento ecológico al desarrollo humano y sistemas sociales (Bronfenbrenner, 1979); la teoria del "empoderamiento" Rappaport (1981); los trabajos en las fortalezas de la familia de Stinnett, Chesser \& DeFrain (1985) y el apoyo social (1981); y finalmente el trabajo realizado por Cronenwett and Brickmant (1982) en practicas de asistencia.

Ahora a través de la investigación y el trabajo profesional, se pueden encontrar una gran variedad de definiciones, con diferentes matices de lo que se pretende decir con intervención centrada en la familia, CANCHILD (Law et al, 2003) nos da una clara definición y experiencia: "la intervención centrada en la familia esta compuesta de un juego de valores, actitudes $\mathrm{y}$ acercamientos en servicios para niños y niñas con necesidades especiales y sus familias. La intervención centrada en la familia reconoce que cada familia es única; que la familia es la constante en la vida de sus hijos e hijas; y que ellos son los expertos en las capacidades y necesidades de sus hijos e hijas. La familia trabaja con profesionales para tomar decisiones informadas, acerca de los servicios y apoyo que el niño/a y la familia recibe. En la intervención centrada en la familia, los profesionales consideran las fortalezas y necesidades de todos los miembros de la familia"(p. 2).

Por otro lado, Espe-Sherwindt (2008) afirma que las practicas centradas en la familia son una filosofía, una manera de entender el cuidado, en el cual, reconoce y respeta el rol central de la familia, "en esencia, las practicas centradas en la familia son una manera sistemática de crear colaboración con las familias (a) tratandolos con respeto y dignidad; (b) entendiendo sus valores y decisiones; y (c) proviendo apoyo para fortalecer $\mathrm{y}$ promocionar su funcionamiento como familia "(p. 136). En otras palabras, los profesionales debemos crear lo que ahora mismo conocemos como 
relaciones cooperativas con las familias, y así poder contribuir en el empoderamiento de esas mismas familias.

El empoderamiento de la familia se entiende como las habilidades que la familia tiene para dirigir de forma eficiente, las diferentes situaciones de su vida, así como, la forma en que asumen el control de los problemas familiares (Dunst, Trivette \& Deal, 1988).

Desde una perspectiva social, el empoderamiento supone varias competencias que la familia ya posee, y si no hay competencias, será posible adquirirlas y será mejor asimilado por la familia a través de su día a día y su ambiente natural (Rappaport, 1981). Cuando trabajamos con familias que están recibiendo servicios de atención temprana, nuestro objetivo debe enfocarse en crear oportunidades para que la familia pueda adquirir el conocimiento y las habilidades necesarias para administrar sus propias necesidades, así pueden promover el bienestar individual y familiar y evitar la dependencia de un profesional.

Los servicios de atención temprana enfocados en la familia, intentan hacer a la familia mas competente en movilizar recursos intra-familiares y extra-familiares , los cuales influyen sobre el niño, los padres y el funcionamiento general de la familia.

Las contribuciones de Zimmerman son agregadas a estas ideas acerca del empoderamiento de las familias. Zimmerman estudio la construcción multidimensional del empoderamiento, a partir del estudio de este constructo, sugiere, que la variación sobre el nivel de empoderamiento individual llevará a cambios en sus dimensiones intrapersonales [p.e.: control percibido, sentido de eficacia, control sobre la motivación, competencia percibida], conciencia critica de la interacción, [entendidos como los agentes causantes, el desarrollo de habilidades, transferencia de habilidades, movilización de recursos] y componentes de comportamiento [p.e.: compromiso comunitario y organizacional, comportamientos copiados] (Zimmerman, 1995).

Por lo tanto, si las practicas centradas en la familia empoderan a la familia, este nivel de empoderamiento debería tener una influencia directa en el estado emocional de los receptores de la intervención y así mismo sobre sus dimensiones intrapersonales, cognitivas e interacciónales (Speer \& Peterson, 2000; Christens, 2012). Este proceso crea un efecto de retroalimentación constante entre el principal objetivo de la intervención (empoderamiento) y el funcionamiento diario de la familia, dando lugar a una influencia positiva en el niño, cuidadores y bienestar familiar.

Evidencias muestran que las practicas de atención temprana centradas en la familia empoderan a la familia (Trivette et al. 2010, Dempsey et al. 2008) y que con el uso de esta filosofía de relación y practica, la familia se empodera mas que con otras practicas donde se ha enfocado solo en el niño.

Tal y como McWilliam (2010) señala, probablemente la idea de proveer atención temprana en ambientes naturales, es la incidencia mas importante que ha pasado en este campo desde los años 90. Familia, escuela y comunidad, son reconocidos como los "medios" que pueden potencialmente contribuir mas en el desarrollo del niño a través de las oportunidades generadas en las rutinas diarias. En este sentido, Work Group on Principles and Practices in Natural Environments (2008) dijo, "la misión de la atención temprana es construir en la familia y proveer apoyo y recursos para ayudar a los miembros de la misma y cuidadores a promover el desarrollo y aprendizaje del niño a través de las oportunidades del día a día". Tener estos aspectos en cuenta es lo que nos da calidad en los servicios de atención temprana.

La investigación debe aclarar algunas de las relaciones entre las diferentes y complejas variables de las practicas de atención temprana y los resultados de familia y niño. Algunos estudios señalan las practicas centradas en la familia como la calidad de los servicios de atención temprana a través de los cuales la familia se habrá empoderado (Dunst, Trivette \& Deal, 1988).

De acuerdo a Derrington, T., Shapiro, B. \& Smith, B. (1999) , "La atención temprana provee una fuente diferente de apoyo social a la familia, la cual reduce el impacto del estrés y estimula la interacción padre e hijo y consecuentemente el desarrollo del niño" (p.23.) De esta y otras reflexiones y con el fin de identificar bajo la percepción de las familias cuales son las practicas que nosotros estamos implementando ahora mismo y cuales serian las practicas ideales o al menos las que las familias desearían tener, McWilliam en 2015 desarrolla la Escala Finesse Family, La cual es una escala de evaluación que incluye diferentes tipos de practicas de intervención temprana, desde un enfoque centrado en las necesidades del niño, a un enfoque sobre las prioridades de la familia en su ambiente natural. Esta escala esta dividida en 19 ítems, los cuales indagan sobre los problemas mas significativos relacionados con las practicas centradas en la familia. Por una parte, la familia evalúa la experiencia típica (p.e.: los servicios comunes que actualmente están recibiendo), y por otra parte, la experiencia ideal (p.e.: los servicios que les gustaría recibir). En ambas, las opciones de respuesta de la familia son a través de la Escala de Likert del 1 al 7, cada puntuación tiene su propio descriptor por practicas recibidas e ideales. La máxima puntuación es 7 ; de ahí que la puntuación máxima corresponderá a una intervención total en prácticas centradas en la familia.

\section{Metodología}

Aquí presentaremos solo una parte de un gran estudio en el cual están participando más de 480 familias de las diferentes comunidades autónomas de España, las cuales tienes hijos e hijas con trastorno del desarrollo o riesgo de padecerlo, y por ello son receptoras de los servicios de atención temprana.

Aquí expondremos el trabajo que se esta realizando para la validación de las escalas de valoración que se están llevando a cabo, empezando en este mismo por el Análisis Factorial Exploratorio de la Escala Finesse Family(McWilliam, 2015).

\section{Participantes}

Los participantes han sido 194 familias españolas con hijos o hijas con trastorno en el desarrollo o riesgo del 
mismo $[56.7 \%$ chicos] y edades comprendidas entre 1 a 144 meses $[\mathrm{M}=51, \mathrm{SD}=18,3]$, los cuales están recibiendo servicios de atención temprana entre 3 y 71 meses $[\mathrm{M}=24, \mathrm{SD}=13,74]$. De los cuales el $66 \%$, también están escolarizados.

\section{Procedimiento y análisis de datos}

Para el procedimiento hemos recolectado toda la Escala de Finesse Family, manteniendo el anonimato de las familias, entregando y recibiendo el dossier del estudio en un sobre en blanco y manteniendo todos los procedimientos requeridos en España para la protección de datos .

Para el analisis de datos, hemos usado SPSS, del cual realizamos el análisis factorial exploratorio (EFA) de los principales componentes con auto valores $>1$, sin la necesidad de forzar los factores para ser extraídos.

Typical Practices: Total variance explained

\begin{tabular}{|c|c|c|c|c|c|c|c|c|c|}
\hline \multirow[t]{2}{*}{ Component } & \multicolumn{3}{|c|}{ Auto initial values } & \multicolumn{3}{|c|}{$\begin{array}{l}\text { Sum of square saturation of } \\
\text { extraction }\end{array}$} & \multicolumn{3}{|c|}{ Sum of square saturations of rotation } \\
\hline & Total & $\begin{array}{lr}\% & \text { of } \\
\text { variance }\end{array}$ & $\begin{array}{l}\% \\
\text { accumulat } \\
\text { ed }\end{array}$ & Total & $\begin{array}{lr}\% & \text { of } \\
\text { variance }\end{array}$ & $\begin{array}{l}\% \\
\text { accumulated }\end{array}$ & Total & $\begin{array}{ll}\% & \text { of } \\
\text { variance }\end{array}$ & $\%$ accumulated \\
\hline 1 & 8,876 & 46,718 & 46,718 & 8,876 & 46,718 & 46,718 & 3,677 & 19,351 & 19,351 \\
\hline 2 & 1,647 & 8,668 & 55,387 & 1,647 & 8,668 & 55,387 & 3,475 & 18,289 & 37,641 \\
\hline 3 & 1,295 & 6,818 & 62,205 & 1,295 & 6,818 & 62,205 & 2,994 & 15,759 & 53,400 \\
\hline 4 & 1,193 & 6,280 & 68,485 & 1,193 & 6,280 & 68,485 & 2,866 & 15,085 & 68,485 \\
\hline 5 &, 866 & 4,559 & 73,043 & & & & & & \\
\hline 6 &, 812 & 4,272 & 77,315 & & & & & & \\
\hline 7 &, 642 & 3,377 & 80,692 & & & & & & \\
\hline 8 & .568 & 2,990 & 83,681 & & & & & & \\
\hline 9 & .501 & 2,638 & 86,319 & & & & & & \\
\hline 10 &, 449 & 2,361 & 88,680 & & & & & & \\
\hline 11 &, 402 & 2,114 & 90,794 & & & & & & \\
\hline 12 & .387 & 2,037 & 92,832 & & & & & & \\
\hline 13 &, 277 & 1,460 & 94,292 & & & & & & \\
\hline 14 &, 256 & 1,347 & 95,638 & & & & & & \\
\hline 15 &, 206 & 1,082 & 96,720 & & & & & & \\
\hline
\end{tabular}

Table 1: Extraction Method: Principal Component Analysis in typical practices.

Ideal Practices: Total variance explained

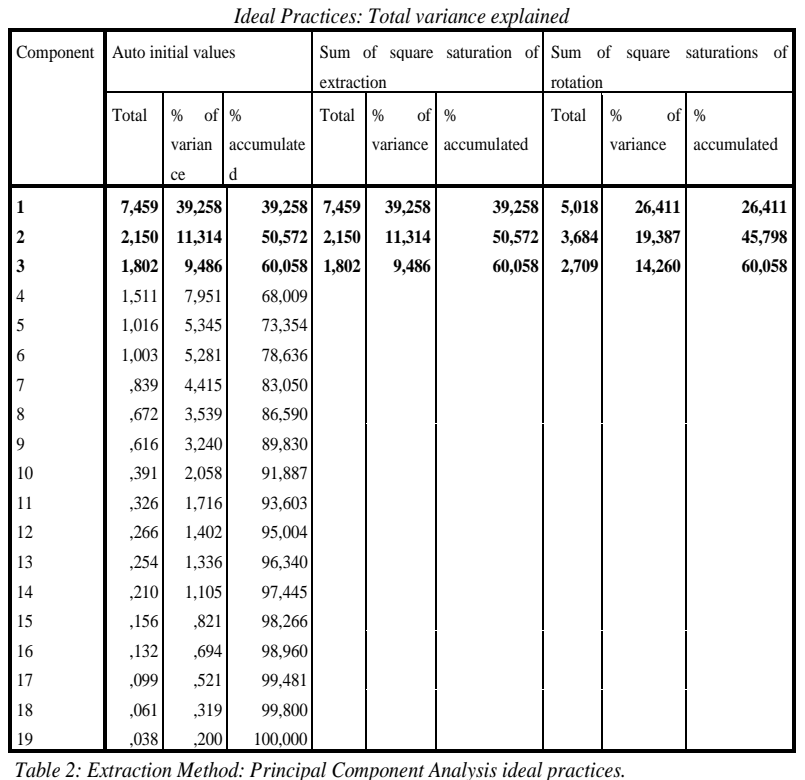

Table 2: Extraction Method: Principal Component Analysis ideal practices.
Hemos utilizado un metodo de rotacion Varimax para ambas tipicas y practicas ideales, y para ser mas restictivos, hemos suprimido la carga por factor de distribución a menos de $(r=.5)$. De este analisis que hemos observado en practicas tipicas (grafico 3) y practicas ideales (grafico 4 ) los siguientes resultados:

Rotated Component Matrix of Typical Practices

\begin{tabular}{|c|c|c|c|c|}
\hline & \multicolumn{4}{|c|}{ Componente } \\
\hline & 1 & 2 & 3 & 4 \\
\hline FF_q15_ET & ,835 & ,131 &, 027 & ,253 \\
\hline FF_q13_ET & ,770 & ,344 &, 008 & ,134 \\
\hline FF_q14_ET & ,604 &, 511 &, 230 & , 136 \\
\hline FF_q11_ET &, 566 & ,224 & ,419 & ,461 \\
\hline FF_q16_ET & ,494 &, 420 & ,403 & ,342 \\
\hline FF_q7_ET &, 145 & ,751 & ,256 & , 193 \\
\hline FF_q4_ET & ,183 & ,749 &, 040 & ,353 \\
\hline FF_q3_ET & ,427 & ,704 &,- 020 & , 170 \\
\hline FF_q19_ET &, 170 &, 567 &, 510 & ,093 \\
\hline FF_q5_ET &, 356 &, 533 &, 359 & ,017 \\
\hline FF_q18_ET &,- 002 & ,456 &, 416 &, 453 \\
\hline FF_q2_ET & ,265 & ,271 & ,705 & ,137 \\
\hline FF_q6_ET &,- 235 &,- 003 & ,704 & , 186 \\
\hline FF_q12_ET &, 530 & , 163 & ,649 & ,200 \\
\hline FF_q17_ET &, 519 & ,312 &, 568 & , 142 \\
\hline FF_q9_ET &, 133 &, 374 & ,086 & ,789 \\
\hline FF_q1_ET & ,087 & , 102 &, 317 & 689 \\
\hline FF_q10_ET &, 432 &,- 006 &, 352 & 644 \\
\hline FF_q8_ET &, 421 & ,259 &,- 068 & ,636 \\
\hline
\end{tabular}

Table 3.Extraction method: Main component analysis. Rotation method: Varimax standardization with Kaiser a. The rotation has converged in 8 iterations.

\begin{tabular}{|c|c|c|c|}
\hline & \multicolumn{3}{|c|}{ Componente } \\
\hline & 1 & 2 & 3 \\
\hline FF_q9_EI & ,829 & & \\
\hline FF_q1_EI & ,786 & & \\
\hline FF_q3_EI & ,746 & ,441 & \\
\hline FF_q11_EI & ,737 & & \\
\hline FF_q10_EI & ,662 & &, 400 \\
\hline FF_q4_EI & ,655 & & \\
\hline FF_q12_EI &, 569 &, 513 & \\
\hline FF_q19_EI &, 557 & & ,468 \\
\hline FF_q8_EI & ,466 & & \\
\hline FF_q13_EI & & ,852 & \\
\hline FF_q16_EI & & ,774 & \\
\hline FF_q18_EI & & ,709 & \\
\hline FF_q15_EI & , 467 & ,666 & \\
\hline FF_q5_EI & &, 547 & \\
\hline FF_q2_EI & & & ,783 \\
\hline FF_q7_EI & & &, 561 \\
\hline FF_q6_EI & & &, 540 \\
\hline FF_q17_EI & & &, 505 \\
\hline FF_q14_EI & ,403 & & ,475 \\
\hline
\end{tabular}

La consistencia de los ítems es luego analizada por el procedimiento de alfa de Cronbach, siendo el total de escala $\alpha=.93$ De acuerdo con los factores que vemos en 
los gráficos anteriores y que será detallado en los resultados.

\section{Resultados}

Como podemos ver en los gráficos anteriores, observamos que en ambas practicas típicas y practicas ideales hay un acuerdo estadístico entre factores. En las practicas típicas podemos claramente distinguir cuatro factores, mientras que en las practicas ideales podemos observar tres factores, detallados a continuación:

\begin{tabular}{llc}
\hline Typical Practices Factors & Cronbach \\
\hline FACT.1 & Intervention and objectives & .84 \\
FACT.2 & Evaluation and programming of the intervention & .77 \\
FACT. 3 & $\begin{array}{l}\text { Transdisciplinarity respecting the holistic vision of } \\
\text { the child }\end{array}$ & .68 \\
FACT. 4 & $\begin{array}{l}\text { Collaborative relationship between professional } \\
\text { and family }\end{array}$ & .73 \\
\hline
\end{tabular}

Table 5. Typical Practices Factors and internal consistency of each of the factors and the full scale in relation to these items

\begin{tabular}{|c|c|c|}
\hline \multicolumn{2}{|c|}{ Ideal Practices Factors } & \multirow{2}{*}{$\begin{array}{c}\text { Cronbach } \\
.83\end{array}$} \\
\hline FACT.1 & $\begin{array}{l}\text { Collaborative relationship between professional } \\
\text { and family }\end{array}$ & \\
\hline FACT.2 & Family's Empowerment & .694 \\
\hline \multirow[t]{2}{*}{ FACT. 3} & Functionality of Early Intervention Practices & .58 \\
\hline & Alpha Cronbach Total Scale & .895 \\
\hline
\end{tabular}

Table 6. Ideal Practices Factors and internal consistency of each of the factors and the full scale in relation to these items

Como podemos ver, podemos definir como futuras líneas de trabajo el estudio sobre el análisis de factor confirmativo de ambas practicas, típicas e ideales.

\section{Conclusiones}

Con el análisis factorial exploratorio (EFA), pretendíamos aclarar las dimensiones interrelacionadas de la Escala Finesse Family (McWilliam, 2015) practicas típicas y/o habituales actualmente llevadas a cabo por los servicios atención temprana, así como las practicas ideales para familias, es decir cuales les gustaría recibir en el futuro en esos mismos servicios.

Como hemos visto anteriormente, podemos concluir que los factores que definen todas las practicas típicas son: a) la intervención y la definición de objetivos; b) la evaluación y la programación de la intervención; c) el equipo transdiciplinar y su visión holística acerca del desarrollo del niño; y d) la relación colaborativa establecida por el profesional y la familia.

Por otro lado, en las practicas ideales, vemos la interrelación de tres factores entre los cuales subrayamos a) relación colaborativa; b) empoderamiento de la familia y c) la funcionabilidad en el día a día de la familia del servicio de atención temprana.

Como vimos en la introducción, estos factores están relacionados con los pilares de las practicas centradas en la familia, por lo tanto, podemos decir que ellos definen la calidad de estos servicios hasta tal punto que estos factores están mas cercanos a enfocarse en la familia y menos en practicas centradas solo en el niño, sus necesidades y déficits.

\section{Referencias}

Allen, R. I., Petr, C. G. (1996). Toward developing standards and measurements for family centered practice in family support programs. En G. H. Singer, L. E. Powers y A. L. Olson (Eds), Redefining family support: innovations in public-private partnerships, 57-85. Baltimore: P.H. Brooks.

Bailey D., Raspa M., Olmsted M., Novak P., Samm M., Humphreys P., Nelson R., Robinson N. Guillen C., (2011). Development and Psychometric Validation of the Family Outcomes Survey-Revised. Journal of Early Intervention, 33(1) 6-29.

Bronfenbrenner, U. (1979). The ecology of human development: Experiments by nature and design. Cambridge, MA: Harvard University Press

Bronfenbrenner, U. \& Morris, P. (1998). The ecology of developmental processes. In R. M. Lerner (Vol. Ed.) \& W. Damon (Series Ed.), Handbook of child psychology, Vol. 1: Theoretical models of human development (5th ed.), 993-1028. New York, NY: Wiley.

Bruder, M.B. (2000). Family-centered early intervention: Clarifying our values for the new millennium. Topics in Early Childhood Special Education, 20(2), 105-122.

Christens, B.D. (2012). Toward relational empowerment. American Journal of community Psicology, 50, 114-128. doi: 10 1007/s10464-011-9483-5.

Cronenwett, L. \& Brickman, P. (1983). Models of helping and coping in childbirth. Nursing Research.32 (2): 84-98

Cunningham, C.C. (2000). Familias de niños con Síndrome de Down. En M.A. Verdugo (Ed.), Familias y Discapacidad Intelectual. Madrid: FEAPS.

Dempsey, I. \& Kenn, D. (2008). A review of processes and Outcomes in Family-Centered servicies for children whit a disability. Topics in Early Childhood Special $\begin{array}{lllll}\text { Education. } & 28 & \text { (1): } & 42-52 & \text { doi: }\end{array}$ 10.1177/0271121408316699.

DeVellis, R.F. (1991). Scale Development: Theory and applications. Newbury Park, CA:Sage

Dunst, C. J. (2007). Early intervention for infants and toddlers with developmental disabilities. In S. L. Odom, R. H. Horner, M. Snell, \& J. Black (Eds.), Handbook of developmental disabilities, 161-180. New York, NY: Guilford Press.

Dunst, C.J., Bruder, M.B., Espe-Sherwindt, M. (2014). Family Capacity - Building in Early Childhood Intervention: Do context ans Stting Matter?. School Community Journal. 21 (1), 37-48

Dunst, C. J., Hamby, D., Trivette, C. M., Raab, M., Bruder, M. B. (2000). Everyday family and community life and children's naturally occurring learning opportunities. Journal of Early Intervention, 23(3), 151-164. doi: 10.1177/10538151000230030501 
Dunst, C.J., Jenkins, V., Trivette, C.M. (1984). Family Support Scale: Reliability and Validity. Journal of individual, Family and Community Wellness, 1, 45-52.

Dunst, C.J.,Trivette, C.M., Deal, A.G. (1988), Enabling and Empowering Families. Cambridge, MA:Brookline Books, 6, 155-157.

Dunst, C. J., Trivette, C. M., Hamby, D. W. (2007). Meta-analysis of family-centered helpgiving practices research. Mental Retardation and Developmental Disabilities Research Reviews, 13, 370-378.

Dunst, C. J. y Trivette, C. M. (1994). What is effective helping. En C. J. Dunst, C. M. Trivette, y A. G. Deal, (Eds), Supporting and strengthening families - Methods, strategies and practices, 162-170.Cambridge: Brookline Books.

Dunst, C.J., Trivette C.M. (1988). Toward experimental evaluation of the Family, Infant and Preschool Program. Hweiss\& F. Jacobs (Eds), Evaluating family programs, 315- 346. New York: Aldine de Gruyter

Dunst, C.J., Trivette, C.M. (2009). Capacity-Building Family-Systems Intervention Practices. Journal of Family Social Work, 12, 119-143.

Dunst, C. J., Trivette, C. M. y LaPointe, N. (1992). Toward clarification of the meaning and key elements of empowerment. Family science review, 5(1/2), 111-130.

Espe-Sherwindt, M. (2008). Family- Centred Practice: Collaboration, Competency and Evidence. Support for Learning, 23(3), 136-143.

FEAPS (2000). Atención Temprana para personas con retraso mental. Orientaciones para la Calidad. Manuales de Buena Práctica. Madrid: FEAPS. Document 55/2000.

Giné, C., Vilaseca, R., Gràcia, M.,García-Dié, M. T. (2004). Early Intervention in Spain: Some Directions for Future Development. Infant and Young Children, 17(3), 236-246.

Gottlieb, B.H. (1981). Social Network and Social Support. Beverly Hills, Calf: Sage Publications.

Grupo de Atención Temprana, GAT. (2000) Libro Blanco de la Atención Temprana. Madrid: Ministerio de Trabajo y Asuntos Sociales. Real Patronato de Prevención y de Atención a Personas con Minusvalía.

INE (2008) Encuesta de Discapacidad, Autonomía Personal y Situaciones de Dependencia (EDAD, 2008). http://www.ine.es/jaxi/menu.do?type=pcaxis\&path=/t15 $/ \mathrm{p} 418 \&$ file $=$ inebase $\& \mathrm{~L}=0$

Krejcie, R.V., Morgan, D.W. (1970). Determining sample size for research activities. Educational and Psychological Measurement, 30, 607-610.

Law, M., Rosenbaum, P., King, G., King, S., Burke-Gaffney, J., Moning, J., Szkut, T., Kertoy, M., Pollock, N., Viscardis, L., \& Teplicky, R. (2003). What is family-centred service? CanChild FCS Sheet \#01. Hamilton, Ontario, Canada: CanChild Centre for Childhood Disability Research, McMaster University. Retrieved January 31, 2015, from https://canchild.ca/system/tenon/assets/attachments/000/ 001/266/original/FCS1.pdf

McWilliam, R.A. (2010). Routines-Based Early Intervention. Supporting Young Children and Their Families. Baltimore, MD: Paul H. Brookes Publishimg.
Nirbhay N. Singh, W. Jonh Curtis, Cynthia R. Ellis, Mary W. Nicholson, Terri M.Villani \& Hollis, A. Wechsler, (1995). Psichometric Analysis of the Family Empowerment Scale . Journal of Emotional and Behavioral Disorders. 3(2) 85-91.

Powell, D. S., Batsche, C. J., Ferro, J., Fox, L. y Dunlap, G. (1997). En Strength-Based Approach in Support of Multi-Risk Families Principles and Issues. Topics in Early Childhood Special Education, 17(1), 1-26.

Rappaport, J. (1981). In Praise of Paradox: A Social Policy of Empowerment Over Prevention, American Journal of Community Psychology, 9 (1) 1-25.

Roggman, L., Boyce, L., Innocenti, M (2008) . Developmental Parenting. A guide for early childhood practitioners. Brookes Publishing. Baltimore

Stinnett, N., Chesser, B., DeFrain, J.D., (1979). Building Family Strengths: blueprints forn accion. Lincoln: University of Nebraska Press.

Trivette, C.M., Dunst, C.J., Hamby, D.W. (2010). Influences of Family Systems Intervention Practices on Parent-child interactions and Child Development. Topics in Early Childhood Special Education. 30 (1) 3-19 doi: $19.1177 / 0271121410364250$

Work Group on Principles and Practices in Natural Environments (2008). Agreed-upon practices for providing early intervention services in natural environments. Retrieve from: http://ectacenter.org/ pdfs/topics/families/Finalmissiona ndprinciples3_11_08.pdf

Zimmerman, M.A.

(1995). Psychological empowerment Issues and Illustrations. American Journal of Community Psychology, 23(5), 581-599. Doi: 10.1007/BF02506983. 